\title{
Mild and Selective Deprotection of tert-Butyl(dimethyl)silyl Ethers with Catalytic Amounts of Sodium Tetrachloroaurate(III) Dihydrate
}

\author{
Qi Zhang*a \\ Xiuqin Kanga \\ Lei Long ${ }^{a}$ \\ Lijuan Zhu ${ }^{\mathrm{a}}$ \\ Yonghai Chai*b \\ a School of Chemistry and Chemical Engineering, Shaanxi \\ Normal University, 199 South Chang'an Road, Xi'an 710062, \\ P. R. of China \\ qiqizhang@snnu.edu.cn \\ ${ }^{b}$ Key Laboratory of Applied Surface and Colloid Chemistry, \\ MOE, Shaanxi Normal University, 199 South Chang'an Road, \\ Xi'an 710062, P. R. of China \\ ychai@snnu.edu.cn
}

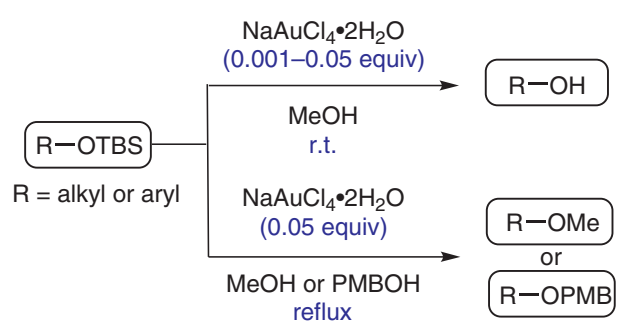

$\mathrm{NaAuCl}_{4} \cdot 2 \mathrm{H}_{2} \mathrm{O}$

(0.001-0.05 equiv)
Received: 04.06.2014

Accepted after revision: 05.08.2014

Published online: 15.09 .2014

DOI: 10.1055/s-0034-1379032; Art ID: ss2014-h0346-op

Abstract A simple and mild method for the removal of tert-butyl(dimethyl)silyl (TBS) protecting groups with catalytic amounts of sodium tetrachloroaurate(III) dihydrate is described. The procedure permits selective deprotection of aliphatic TBS ethers in good to excellent yields in the presence of aromatic TBS ethers, aliphatic triisopropylsilyl ethers, aliphatic tert-butyl(diphenyl)silyl ethers, or sterically hindered aliphatic TBS ethers. Additionally, TBS ethers can also be transformed into 4-methoxybenzyl ethers or methyl ethers in one pot by using larger quantities of the catalyst and a higher reaction temperature.

Key words deprotection, silyl ethers, alcohols, ethers, catalysis, gold

Protection/deprotection strategies play important roles in modern organic synthesis. ${ }^{1,2}$ The tert-butyl(dimethyl)silyl (TBS) group is one of the most widely used protecting groups for alcohols because of its easy installation, its stability to various reaction conditions, and the selectivity of its cleavage reaction. Numerous methods are available for removal of TBS groups, ${ }^{3-9}$ including the use of acidic, ${ }^{4}$ basic, $^{5}$ reducing, ${ }^{6}$ oxidizing, ${ }^{7}$ or fluoride-based reagents ${ }^{8}$ among others. ${ }^{9}$ However, new mild and selective protocols for the deprotection of TBS ethers are still in great demand for use in syntheses of multifunctional compounds, particularly complex natural products.

Commercially available sodium tetrachloroaurate(III) dihydrate $\left(\mathrm{NaAuCl}_{4} \cdot 2 \mathrm{H}_{2} \mathrm{O}\right)$ is the least expensive gold catalyst and has been used in several types of reaction, including nucleophilic addition to multiple bonds, ${ }^{10-13}$ nucleophilic substitution of propargylic alcohols, ${ }^{14,15}$ nonsymmetrical etherization, ${ }^{16}$ and others. ${ }^{17,18}$ In the course of an ongoing total-synthesis project, we serendipitously found that the TBS protecting group was cleanly removed in the presence of a small amount of $\mathrm{NaAuCl}_{4} \cdot 2 \mathrm{H}_{2} \mathrm{O}$. Inspired by this observation, we explored the possibility of using NaAu$\mathrm{Cl}_{4} \cdot 2 \mathrm{H}_{2} \mathrm{O}$ as an effective catalyst for the deprotection of TBS ethers.
Table 1 Optimization of Conditions for the Sodium Tetrachloroaurate(III) Catalyzed Deprotection of TBS Ether 1

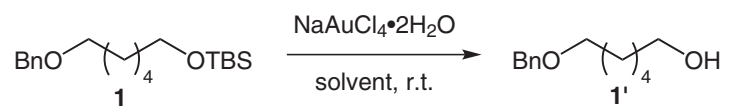

\begin{tabular}{lllll}
\hline Entry & Catalyst $\left(\right.$ equiv $\left.^{\mathrm{a}}\right)$ & Solvent & Time $(\mathrm{h})$ & Yield $^{\mathrm{b}}(\%)$ \\
\hline 1 & 0.01 & $\mathrm{MeOH}$ & 3.5 & 95 \\
2 & 0.01 & THF & 12 & 73 \\
3 & 0.01 & $\mathrm{MeCN}$ & 12 & 53 \\
4 & 0.01 & acetone- $\mathrm{H}_{2} \mathrm{O}(1: 1 \mathrm{v} / \mathrm{v})$ & 12 & 40 \\
5 & 0.005 & $\mathrm{MeOH}$ & 7 & 96 \\
6 & 0.001 & $\mathrm{MeOH}$ & 12 & 95 \\
7 & 0.0005 & $\mathrm{MeOH}$ & 36 & 93 \\
8 & 0.0001 & $\mathrm{MeOH}$ & 36 & $25\left(72^{\mathrm{c}}\right)$ \\
\hline
\end{tabular}

a With respect to the substrate 1 .

$\mathrm{b}$ Isolated yield of pure product.

' Recovery of substrate $\mathbf{1 .}$

First, we evaluated the effects of the solvent and the catalyst loading on the gold(III)-catalyzed desilylation of the TBS ether of 6-(benzyloxy)hexan-1-ol (1) (Table 1). In the presence of 0.01 equivalents of sodium tetrachloroaurate(III) in methanol, deprotection of the TBS ether 1 proceeded smoothly to give the corresponding alcohol 1' in 95\% yield after 3.5 hours at room temperature (Table 1, entry 1 ). The cleavage also proceeded in other polar solvents, but yields were much lower (entries 2-4). Alcohol 1' was still obtained in excellent yields and in reasonable reaction times when the amount of catalyst was reduced to 0.005 , 0.001 , or even 0.0005 equivalents, (entries $5-7$ ), but the desilylation became sluggish when 0.0001 equivalents of the catalyst were used (entry 8).

Next, we synthesized various aliphatic and aromatic TBS ethers to examine the substrate scope of our deprotection method. The deprotection reaction of the primary TBS 
ethers was conducted in the presence of 0.001-0.005 equivalents of sodium tetrachloroaurate(IIII) dihydrate in methanol at room temperature. Primary TBS ethers containing electron-donating or electron-withdrawing groups were readily deprotected to give the corresponding alcohols in high yields (Table 2, entries 1-5). Secondary and tertiary TBS ethers were also desilylated smoothly, although longer reaction times or greater catalyst loadings were required (entries 6 and 7). Although sodium tetrachloroaurate(IIII) dihydrate catalyzes addition reactions of alkenes or alkynes, ${ }^{10-13}$ the TBS protecting group of compound $\mathbf{9}$ was removed successfully under the current condition without any effect on the double bond (entry 8). Aromatic TBS ethers are usually deprotected by treatment with basic re- agents or fluoride-based reagents that frequently produce unwanted side reactions, such as silyl migration. ${ }^{19}$ Gratifyingly, the cleavage of aromatic TBS ethers proceeded well in the presence of $0.05-0.1$ equivalents of the catalyst in methanol at room temperature. Aromatic TBS ethers bearing electron-donating groups were much more reactive than those bearing electron-withdrawing groups (entries 9-12).

Next, we examined the selective deprotection of TBS ethers containing various sensitive functional groups under the optimized conditions (Table 3). On treatment with 0.005 equivalents of sodium tetrachloroaurate(III) dihydrate in methanol at room temperature, the TBS group in substrates 14-18 was selectively removed, whereas other

Table 2 Deprotection of Primary, Secondary, Tertiary and Aryl TBS Ethers Catalyzed by Sodium Tetrachloroaurate(III) in Methanol

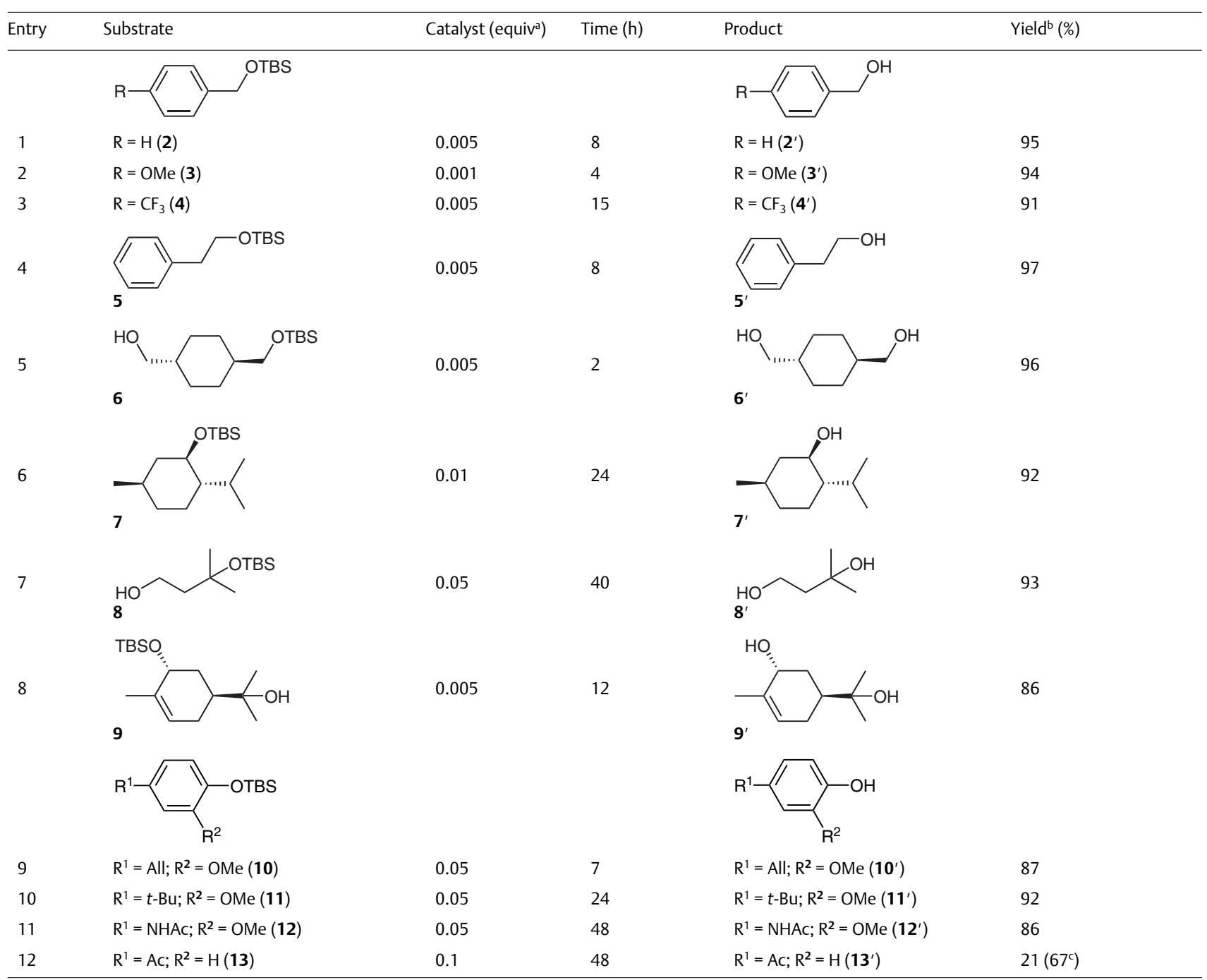

${ }^{a}$ With respect to the substrate.

${ }^{b}$ Isolated yield of pure product.

' Recovery of substrate 13. 
Synthesis

Q. Zhang et al.

Table 3 Selective Deprotection of Various Silyl Ethers Catalyzed by Sodium Tetrachloroaurate(III) in Methanol

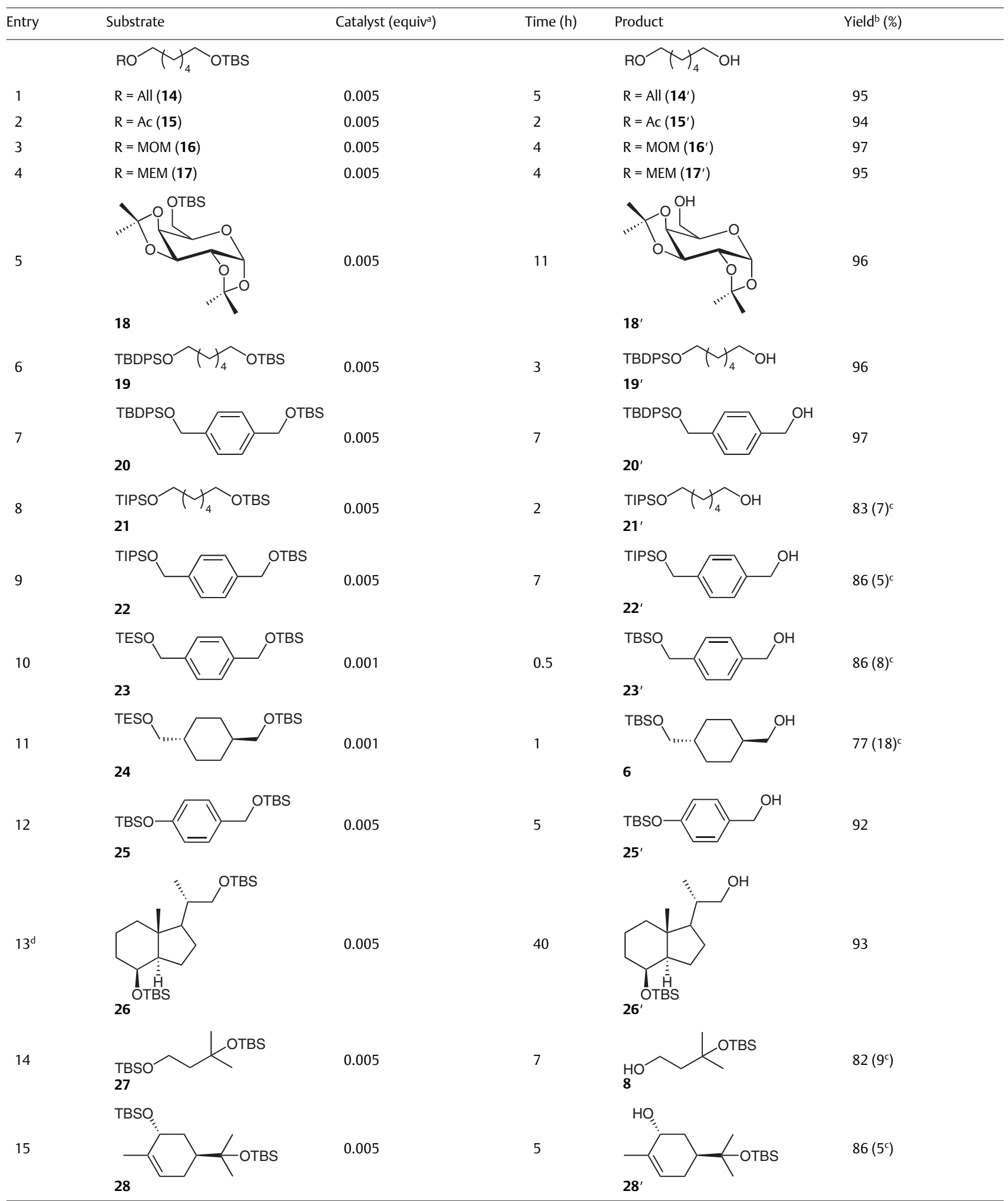

With respect to the substrate.

${ }^{\mathrm{b}}$ Isolated yield of pure product.

' Yield of diol.

d EtOAc-MeOH (1:1, v/v) was used as the solvent. 
common acid-labile protecting groups such as allyl, acetyl, methoxymethyl, (2-methoxyethoxy)methyl, and isopropylidene were unaffected (Table 3, entries 1-5). The catalyst also showed good selectivity to various silyl protecting groups. Preferential cleavage of TBS ether groups in the presence of tert-butyl(diphenyl)silyl (TBDPS) ether groups gave the monodeprotected products in high yields, and the corresponding diols were not obtained (entries 6 and 7). When a less-bulky triisopropylsilyl group (compared with TBDPS) and a TBS groups were present together, the deprotection was less selective, but the desired monoalcohols were still obtained as the major products ( $\geq 83 \%$ yield) (entries 8 and 9). Moderate selectivity between triethylsilyl and TBS protecting groups was achieved by using 0.001 equivalents of the catalyst (entries 10 and 11). An aliphatic TBS ether group was selectively removed in the presence of an aromatic TBS ether group in 92\% yield (entry 12). We also examined the possibility of selectively deprotecting TBS diethers (entries 13-15). In all cases, the less-hindered TBS ether group was cleaved in preference to the morehindered one in good to excellent yield; this makes our method very useful in total syntheses of complicated compounds such as natural products or their analogues.

Because nonsymmetrical ethers can be prepared from alcohols by using sodium tetrachloroaurate(III) dihydrate as catalyst, ${ }^{16}$ we decided to examine the one-pot transformation of TBS ethers into other frequently used ethers. Under relatively harsh condition [ $\mathrm{NaAuCl}_{4} \cdot 2 \mathrm{H}_{2} \mathrm{O}$ (0.05 equiv), THF, reflux], TBS ether 1 reacted with 4-methoxybenzyl alcohol to give the desired ether $\mathbf{2 9}$ directly (Scheme 1). More interestingly, substrate 30, which readily forms the corresponding carbocation, gave either the deprotected product $30^{\prime}$ or the methyl ether 31, depending on the amount of the catalyst used and the reaction temperature (Scheme 1).

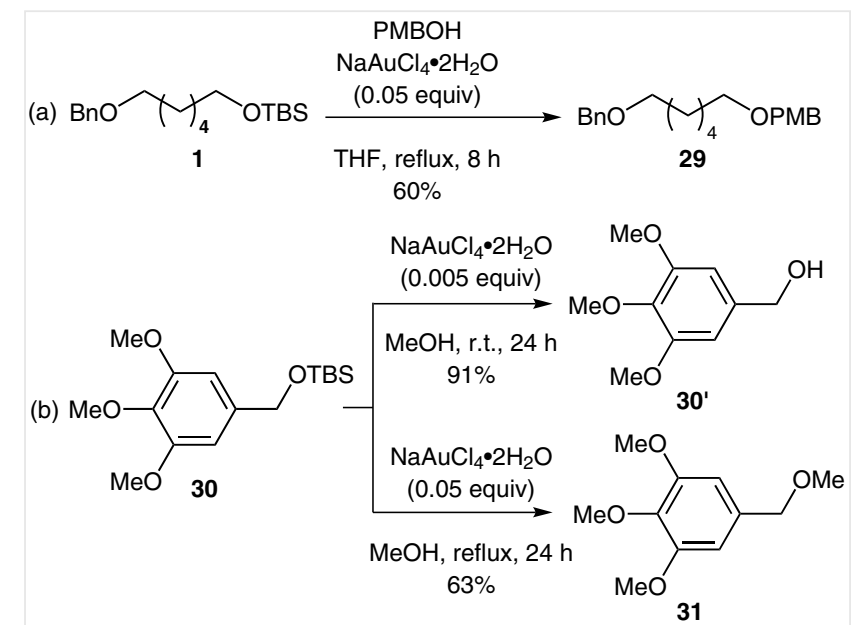

Scheme 1 Transformation of TBS ethers into 4-methoxybenzyl or methyl ethers
In conclusion, we have developed an effective protocol for the deprotection of TBS ethers by using a very small amount of sodium tetrachloroaurate(III) dihydrate as catalyst. Notable features of the protocol include mild conditions, low cost, easy operations, good functional group compatibility, and high selectivity. The method should therefore have widespread applications in syntheses of complex, multifunctional, or sensitive molecules. In addition, by using larger amounts of catalyst and higher reaction temperatures, TBS ethers can also be transformed into 4-methoxybenzyl or methyl ethers in a one-pot process.

${ }^{1} \mathrm{H}$ NMR and ${ }^{13} \mathrm{C}$ NMR spectra were recorded in $\mathrm{CDCl}_{3}$ or $\mathrm{CD}_{3} \mathrm{OD}$ on a Bruker Avance 300 or Bruker Avance 400 instrument. Chemical shifts

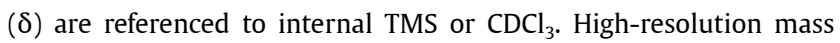
spectra were recorded on a Bruker maXis Impact mass spectrometer. Melting points were determined by using a Stuart Scientific SMP10 instrument and are uncorrected. IR spectra were recorded in the ATR mode on a Nicolet 6700 FT-IR Thermo Scientific spectrometer; only the more significant peaks are reported. All reagents and solvents obtained commercially and were used as received without further purification. Reactions were monitored by TLC on glass-backed plates coated with a $0.2 \mathrm{~mm}$ thickness of silica gel 60 F254; chromatograms were visualized by UV radiation $(254 \mathrm{~nm})$ or by staining with phosphomolybdic acid and $\mathrm{H}_{2} \mathrm{SO}_{4}$. Column chromatography was performed on 300-400 mesh silica gel.

Except for compound $\mathbf{8}, \mathbf{2 8}$, and $\mathbf{3 0}$, all TBS ethers were prepared according to the procedures reported in the literature.

\section{3-\{[tert-Butyl(dimethyl)silyl]oxy\}-3-methylbutan-1-ol (8)}

$\mathrm{NaAuCl}_{4} \cdot 2 \mathrm{H}_{2} \mathrm{O}$ (4.0 mg, $0.01 \mathrm{mmol}, 0.005$ equiv) was added to a solution of disilyl ether $\mathbf{2 7}^{20}$ (665 mg, $2 \mathrm{mmol}$ ) in $\mathrm{MeOH}(4 \mathrm{~mL})$ at r.t., and the mixture was stirred at r.t. for $7 \mathrm{~h}$. The mixture was then diluted with EtOAc $(10 \mathrm{~mL})$, filtered through activated alumina, and concentrated in vacuo. The residue was purified by flash column chromatography [silica gel, EtOAc-PE (1:5)] to give a colorless oil; yield: $358 \mathrm{mg}$ (82\%).

IR (KBr): 3355, 2945, 2857, 1468, 1250, $1041 \mathrm{~cm}^{-1}$.

${ }^{1} \mathrm{H}$ NMR $\left(300 \mathrm{MHz}, \mathrm{CDCl}_{3}\right): \delta=3.82(\mathrm{t}, J=5.9 \mathrm{~Hz}, 2 \mathrm{H}), 1.72(\mathrm{t}, J=5.9$ $\mathrm{Hz}, 2 \mathrm{H}), 1.31$ (s, $6 \mathrm{H}), 0.87$ (s, $9 \mathrm{H}), 0.13$ (s, $6 \mathrm{H})$.

${ }^{13} \mathrm{C} \mathrm{NMR}\left(75 \mathrm{MHz}, \mathrm{CDCl}_{3}\right): \delta=75.31,60.00,45.70,29.86,25.79,17.90$, -2.01 .

MS (ESI, MeOH): $m / z=241[\mathrm{M}+\mathrm{Na}]^{+}$.

HRMS-ESI: $m / z$ [M $+\mathrm{Na}]^{+}$Calcd for $\mathrm{C}_{11} \mathrm{H}_{26} \mathrm{NaO}_{2} \mathrm{Si}$ : 241.1600; found: 241.1612.

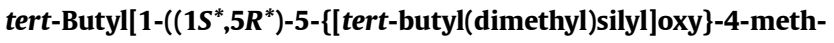
ylcyclohex-3-en-1-yl)-1-methylethoxy]dimethylsilane (28)

TBSOTf (1.52 mL, $6.6 \mathrm{mmol}$ ) was added dropwise to a solution of diol $\mathbf{9}^{\mathbf{2} 1}$ (511 $\mathrm{mg}, 3 \mathrm{mmol}$ ) and 2,6-lutidine $(0.76 \mathrm{~mL}, 6.6 \mathrm{mmol}$ ) in dry $\mathrm{CH}_{2} \mathrm{Cl}_{2}(6 \mathrm{~mL})$ at $0{ }^{\circ} \mathrm{C}$, and the mixture was stirred for $5 \mathrm{~h} . \mathrm{H}_{2} \mathrm{O}(5 \mathrm{~mL})$ and $\mathrm{CH}_{2} \mathrm{Cl}_{2}(20 \mathrm{~mL})$ were added, and the organic layer was separated and washed successively with sat. aq $\mathrm{NaHCO}_{3}(5 \mathrm{~mL}), \mathrm{H}_{2} \mathrm{O}(2 \times 5 \mathrm{~mL})$, and brine $(5 \mathrm{~mL})$, then dried $\left(\mathrm{MgSO}_{4}\right)$ and filtered. The filtrate was concentrated in vacuo and the resulting residue was purified by flash column chromatography [silica gel, EtOAc-PE (1:25)] to give a colorless oil; yield: $1.065 \mathrm{~g}(89 \%)$. 
IR (KBr): 2966, 2920, 2861, 1475, 1368, $1256 \mathrm{~cm}^{-1}$.

${ }^{1} \mathrm{H} \mathrm{NMR}\left(400 \mathrm{MHz}, \mathrm{CDCl}_{3}\right): \delta=5.52(\mathrm{dd}, J=3.6,1.6 \mathrm{~Hz}, 1 \mathrm{H}), 4.01(\mathrm{br} \mathrm{s}$, $1 \mathrm{H}), 2.11(\mathrm{dt}, J=16.8,5.6 \mathrm{~Hz}, 1 \mathrm{H}), 1.88-1.78(\mathrm{~m}, 2 \mathrm{H}), 1.75-1.67(\mathrm{~m}$, $4 \mathrm{H}), 1.37(\mathrm{dt}, J=3.6,13.2 \mathrm{~Hz}, 1 \mathrm{H}), 1.21(\mathrm{~s}, 3 \mathrm{H}), 1.18(\mathrm{~s}, 3 \mathrm{H}), 0.91(\mathrm{~s}, 9$ $\mathrm{H}), 0.85(\mathrm{~s}, 9 \mathrm{H}), 0.10(\mathrm{~s}, 6 \mathrm{H}), 0.07(\mathrm{~s}, 6 \mathrm{H})$.

${ }^{13} \mathrm{C}$ NMR $\left(75 \mathrm{MHz}, \mathrm{CDCl}_{3}\right): \delta=134.45,125.05,74.73,69.46,39.62$, $33.58,28.37,27.57,26.86,25.95,25.95,21.25,18.29,18.15,-2.02$ (2 C).

MS (ESI, $\mathrm{MeOH}): m / z=421[\mathrm{M}+\mathrm{Na}]^{+}$.

HRMS-ESI: $m / z[\mathrm{M}+\mathrm{Na}]^{+}$calcd for $\mathrm{C}_{22} \mathrm{H}_{46} \mathrm{NaO}_{2} \mathrm{Si}_{2}: 421.2934$; found: 421.2940 .

\section{tert-Butyl(dimethyl)[(3,4,5-trimethoxybenzyl)oxy]silane (30)}

A solution of alcohol $\mathbf{3 0}^{\prime 22}$ (396 mg, $2 \mathrm{mmol}$ ), imidazole (300 mg, 4.4 $\mathrm{mmol})$, and $\mathrm{TBSCl}(332 \mathrm{mg}, 2.2 \mathrm{mmol})$ in anhyd $\mathrm{CH}_{2} \mathrm{Cl}_{2}(4 \mathrm{~mL})$ was stirred at overnight at r.t. $\mathrm{H}_{2} \mathrm{O}(5 \mathrm{~mL})$ and $\mathrm{CH}_{2} \mathrm{Cl}_{2}(20 \mathrm{~mL})$ were added to the mixture, and the organic layer was separated, washed successively with sat. aq $\mathrm{NaHCO}_{3}(5 \mathrm{~mL}), \mathrm{H}_{2} \mathrm{O}(2 \times 5 \mathrm{~mL})$, and brine $(5 \mathrm{~mL})$, then dried $\left(\mathrm{MgSO}_{4}\right)$ and filtered. The filtrate was concentrated in vacuo and the residue was purified by flash column chromatography [silica gel, EtOAc-PE (1:25)] to give a colorless oil; yield: $569 \mathrm{mg}$ (91\%).

IR (KBr): 2962, 2928, 2857, 1596, 1499, 1459, 1378, 1017, 832, 775 $\mathrm{cm}^{-1}$.

${ }^{1} \mathrm{H}$ NMR $\left(300 \mathrm{MHz}, \mathrm{CDCl}_{3}\right): \delta=6.57$ (s, $\left.2 \mathrm{H}\right), 4.69$ (s, $\left.2 \mathrm{H}\right), 3.85(\mathrm{~s}, 6 \mathrm{H})$, $3.83(\mathrm{~s}, 3 \mathrm{H}), 0.96(\mathrm{~s}, 9 \mathrm{H}), 0.11(\mathrm{~s}, 6 \mathrm{H})$.

${ }^{13} \mathrm{C}$ NMR $\left(75 \mathrm{MHz}, \mathrm{CDCl}_{3}\right): \delta=153.16,137.14,136.78,102.80,64.86$, $60.78,55.96,25.89,18.36,-5.28$.

MS (ESI, $\mathrm{MeOH}): m / z=335[\mathrm{M}+\mathrm{Na}]^{+}$.

HRMS-ESI: $m / z$ [M $+\mathrm{Na}]^{+}$calcd for $\mathrm{C}_{16} \mathrm{H}_{28} \mathrm{NaO}_{4} \mathrm{Si}$ : 335.1655; found: 335.1663.

\section{Deprotection of TBS Ethers; General Procedure}

A solution of the TBS ether $(2 \mathrm{mmol})$ in $\mathrm{MeOH}(4 \mathrm{~mL})$ was treated with $\mathrm{NaAuCl}_{4} \cdot 2 \mathrm{H}_{2} \mathrm{O}(4.0 \mathrm{mg}, 0.01 \mathrm{mmol}, 0.005$ equiv $)$ at r.t. When the starting material had disappeared (TLC), mixture was diluted with EtOAc $(10 \mathrm{~mL})$ and filtered through activated alumina. The solution was then concentrated in vacuo and the resulting residue was purified by flash column chromatography.

\section{6-(Benzyloxy)hexan-1-ol (1' $)^{4 \mathrm{n}}$}

Prepared as a colorless oil from silyl ether $\mathbf{1}^{4 \mathrm{n}}$ according to the general procedure using $0.01-0.0001$ equiv of $\mathrm{NaAuCl}_{4} \cdot 2 \mathrm{H}_{2} \mathrm{O}$ in various solvents; for yields, see Table 1 .

${ }^{1} \mathrm{H}$ NMR (300 MHz, $\mathrm{CDCl}_{3}$ ): $\delta=7.34-7.33(\mathrm{~m}, 5 \mathrm{H}), 4.50(\mathrm{~s}, 2 \mathrm{H}), 3.63$ $(\mathrm{t}, J=6.5 \mathrm{~Hz}, 2 \mathrm{H}), 3.47(\mathrm{t}, J=6.5 \mathrm{~Hz}, 2 \mathrm{H}), 1.66-1.55(\mathrm{~m}, 4 \mathrm{H}), 1.40-$ $1.38(\mathrm{~m}, 4 \mathrm{H}), 1.26$ (br s, $1 \mathrm{H})$.

${ }^{13} \mathrm{C}$ NMR $\left(75 \mathrm{MHz}, \mathrm{CDCl}_{3}\right): \delta=138.64,128.31,127.60,127.46,72.86$, 70.31, 62.87, 32.68, 29.68, 25.98, 25.55.

MS (ESI, $\mathrm{MeOH}): m / z=231[\mathrm{M}+\mathrm{Na}]^{+}$.

HRMS-ESI: $m / z[M+N a]^{+}$calcd for $\mathrm{C}_{13} \mathrm{H}_{20} \mathrm{NaO}_{2}: 231.1361$; found: 231.1351 .

\section{Benzyl Alcohol (2') ${ }^{4 b}$}

Prepared according to the general procedure from $2^{4 \mathrm{~b}}$ (445 mg), and purified by flash column chromatography [silica gel, EtOAc-PE (1:3)] as a colorless oil; yield: $206 \mathrm{mg}$ (95\%).
${ }^{1} \mathrm{H}$ NMR (300 MHz, $\left.\mathrm{CDCl}_{3}\right): \delta=7.37-7.28(\mathrm{~m}, 5 \mathrm{H}), 4.69(\mathrm{~s}, 2 \mathrm{H}), 1.69$ (br s, $1 \mathrm{H}$ ).

${ }^{13} \mathrm{C} \mathrm{NMR}\left(75 \mathrm{MHz}, \mathrm{CDCl}_{3}\right)$ : $\delta=140.83,128.46,127.52,126.91,65.13$. MS (ESI, MeOH): $m / z=131[\mathrm{M}+\mathrm{Na}]^{+}$.

HRMS-ESI: $m / z[\mathrm{M}+\mathrm{Na}]^{+}$calcd for $\mathrm{C}_{7} \mathrm{H}_{8} \mathrm{NaO}$ : 131.0473; found: 131.0478 .

\section{(4-Methoxyphenyl)methanol ( $\left.3^{\prime}\right)^{4 \mathrm{j}}$}

$\mathrm{NaAuCl}_{4} \cdot 2 \mathrm{H}_{2} \mathrm{O}(0.8 \mathrm{mg}, 0.002 \mathrm{mmol}, 0.001$ equiv) was added to a solution of silyl ether $3^{4 \mathrm{j}}(505 \mathrm{mg}, 2 \mathrm{mmol})$ in $\mathrm{MeOH}(4 \mathrm{~mL})$ at r.t., and the mixture was stirred at r.t. for $4 \mathrm{~h}$. The mixture was then diluted with EtOAc $(10 \mathrm{~mL})$, and filtered through activated alumina. The solution was concentrated in vacuo and resulting residue was purified by flash column chromatography [silica gel, EtOAc-PE (1:3)] to give a colorless oil; yield: $260 \mathrm{mg}$ (94\%).

${ }^{1} \mathrm{H}$ NMR $\left(300 \mathrm{MHz}, \mathrm{CDCl}_{3}\right): \delta=7.27(\mathrm{~d}, J=8.7 \mathrm{~Hz}, 2 \mathrm{H}), 6.88(\mathrm{~d}, J=8.7$ $\mathrm{Hz}, 2 \mathrm{H}), 4.46$ (s, $2 \mathrm{H}), 3.80$ (s, $3 \mathrm{H})$.

${ }^{13} \mathrm{C}$ NMR $\left(75 \mathrm{MHz}, \mathrm{CDCl}_{3}\right): \delta=159.11,130.42,129.27,113.70,71.37$, 55.12 .

MS (ESI, MeOH): $m / z=161[\mathrm{M}+\mathrm{Na}]^{+}$.

HRMS-ESI: $m / z[M+N a]^{+}$calcd for $\mathrm{C}_{8} \mathrm{H}_{10} \mathrm{NaO}_{2}: 161.0578$; found: 161.0597.

\section{[4-(Trifluoromethyl)phenyl]methanol $\left(4^{\prime}\right)^{23}$}

Prepared according to the general procedure from silyl ether $\mathbf{4}^{24}$ (581 $\mathrm{mg}$ ), and purified by flash column chromatography [silica gel, EtOAcPE (1:5)] as a colorless oil; yield: $321 \mathrm{mg}(91 \%)$.

${ }^{1} \mathrm{H}$ NMR $\left(300 \mathrm{MHz}, \mathrm{CDCl}_{3}\right): \delta=7.62(\mathrm{~d}, J=8.1 \mathrm{~Hz}, 2 \mathrm{H}), 7.47(\mathrm{~d}, J=8.1$ $\mathrm{Hz}, 2 \mathrm{H}$ ), 4.77 (s, $2 \mathrm{H}), 1.91$ (br s, $1 \mathrm{H}$ ).

${ }^{13} \mathrm{C}$ NMR (75 MHz, $\mathrm{CDCl}_{3}$ ): $\delta=145.67,129.76(\mathrm{q}), 126.80,125.42(\mathrm{q})$, 122.78, 64.40 .

MS (ESI, MeOH): $m / z=199[\mathrm{M}+\mathrm{Na}]^{+}$.

HRMS-ESI: $m / z[\mathrm{M}+\mathrm{Na}]^{+}$calcd for $\mathrm{C}_{8} \mathrm{H}_{7} \mathrm{~F}_{3} \mathrm{NaO}$ : 199.0347; found: 199.0342 .

\section{2-Phenylethanol $\left(5^{\prime}\right)^{8 a}$}

Prepared according to the general procedure from silyl ether $\mathbf{5}^{8 \mathrm{a}}$ (473 $\mathrm{mg}$ ), and purified by flash column chromatography [silica gel, EtOAcPE (1:3)] as a colorless oil; yield: $237 \mathrm{mg}(97 \%)$.

${ }^{1} \mathrm{H} \mathrm{NMR}\left(300 \mathrm{MHz}, \mathrm{CDCl}_{3}\right): \delta=7.33-7.30(\mathrm{~m}, 2 \mathrm{H}), 7.24-7.22(\mathrm{~m}, 3 \mathrm{H})$, $3.86(\mathrm{t}, J=5.0 \mathrm{~Hz}, 2 \mathrm{H}), 2.87(\mathrm{t}, J=5.0 \mathrm{~Hz}, 2 \mathrm{H}), 1.52(\mathrm{br} \mathrm{s}, 1 \mathrm{H})$.

${ }^{13} \mathrm{C}$ NMR $\left(75 \mathrm{MHz}, \mathrm{CDCl}_{3}\right): \delta=138.48,128.97,128.51,126.40,63.58$, 39.14.

MS (ESI, MeOH): $m / z=145[\mathrm{M}+\mathrm{Na}]^{+}$.

HRMS-ESI: $m / z[\mathrm{M}+\mathrm{Na}]^{+}$calcd for $\mathrm{C}_{8} \mathrm{H}_{10} \mathrm{NaO}: 145.0629$; found: 145.0630 .

( \pm )-trans-Cyclohexane-1,4-diyldimethanol $\left(6^{\prime}\right)^{25}$

Prepared according to the general procedure from $6^{26}(517 \mathrm{mg})$, and purified by flash column chromatography [silica gel, EtOAc-PE (3:1)] as a white solid; yield: $277 \mathrm{mg}(96 \%) ; \mathrm{mp} 63-65^{\circ} \mathrm{C}$.

${ }^{1} \mathrm{H} \mathrm{NMR}\left(300 \mathrm{MHz}, \mathrm{CDCl}_{3}\right): \delta=3.47(\mathrm{~d}, J=4.8 \mathrm{~Hz}, 4 \mathrm{H}), 1.86-1.84(\mathrm{~m}, 4$ $\mathrm{H}), 1.46-1.44[\mathrm{~m}, 4 \mathrm{H}$, including $1.46(\mathrm{br} \mathrm{s}, 2 \mathrm{H})], 1.01-0.96(\mathrm{~m}, 4 \mathrm{H})$.

${ }^{13} \mathrm{C} \mathrm{NMR}\left(75 \mathrm{MHz}, \mathrm{CDCl}_{3}\right): \delta=68.51,40.57,28.87$.

MS (ESI, MeOH): $m / z=167[\mathrm{M}+\mathrm{Na}]^{+}$. 
HRMS-ESI: $m / z$ [M $+\mathrm{Na}]^{+}$calcd for $\mathrm{C}_{8} \mathrm{H}_{16} \mathrm{NaO}_{2}:$ 167.1048; found: 167.1039.

\section{$(\boldsymbol{R})$-(-)-Menthol ( $\left.\mathbf{7}^{\prime}\right)^{8 \mathrm{~b}}$}

$\mathrm{NaAuCl}_{4} \cdot 2 \mathrm{H}_{2} \mathrm{O}$ ( $8.0 \mathrm{mg}, 0.02 \mathrm{mmol}, 0.01$ equiv) was added to a solution of silyl ether $7^{8 \mathrm{~b}}$ (541 mg, $2 \mathrm{mmol}$ ) in $\mathrm{MeOH}(4 \mathrm{~mL})$ at r.t., and the mixture was stirred at r.t. for $24 \mathrm{~h}$. The mixture was then diluted with EtOAc $(10 \mathrm{~mL})$, filtered through activated alumina, and concentrated in vacuo. The residue was purified by flash column chromatography [silica gel, EtOAc-PE (1:5)] to give a white solid; yield: $288 \mathrm{mg}$ (92\%); mp $42-43^{\circ} \mathrm{C}$.

${ }^{1} \mathrm{H}$ NMR (300 MHz, $\left.\mathrm{CDCl}_{3}\right): \delta=3.41$ (br s, $\left.1 \mathrm{H}\right), 2.21-2.13(\mathrm{~m}, 1 \mathrm{H}$ ), 1.99-1.95 (m, $1 \mathrm{H}), 1.64-1.58$ ( $\mathrm{m}, 2 \mathrm{H}), 1.43-1.38$ (m, $1 \mathrm{H}), 1.28-1.25$ (m, $2 \mathrm{H}), 1.11-1.08(\mathrm{~m}, 1 \mathrm{H}), 1.00-0.85(\mathrm{~m}, 1 \mathrm{H}), 0.93(\mathrm{~d}, J=5.1 \mathrm{~Hz}, 3$ $\mathrm{H}), 0.92(\mathrm{~d}, J=4.8 \mathrm{~Hz}, 3 \mathrm{H}), 0.81(\mathrm{~d}, J=5.1 \mathrm{~Hz}, 3 \mathrm{H})$.

${ }^{13} \mathrm{C}$ NMR $\left(75 \mathrm{MHz}, \mathrm{CDCl}_{3}\right.$ ): $\delta=71.53,50.16,45.08,34.55,31.63,25.85$, 23.19, 22.17, 20.96, 16.11 .

MS (ESI, MeOH): $m / z=179[\mathrm{M}+\mathrm{Na}]^{+}$.

HRMS-ESI: $m / z$ [M $+\mathrm{Na}]^{+}$calcd for $\mathrm{C}_{10} \mathrm{H}_{20} \mathrm{NaO}$ : 179.1412; found: 179.1421.

\section{3-Methylbutane-1,3-diol (8') ${ }^{27}$}

$\mathrm{NaAuCl}_{4} \cdot 2 \mathrm{H}_{2} \mathrm{O}$ (39.8 mg, $0.1 \mathrm{mmol}, 0.05$ equiv) was added to a solution of silyl ether 8 (437 mg, $2 \mathrm{mmol}$ ) in $\mathrm{MeOH}(4 \mathrm{~mL})$ at r.t., and the mixture was stirred at r.t. for $40 \mathrm{~h}$. The mixture was then diluted with EtOAc (10 mL), filtered through activated alumina, and concentrated in vacuo. The residue was purified by flash column chromatography [silica gel, EtOAc-PE (2:1)] to give a colorless oil; yield: $194 \mathrm{mg}$ (93\%). ${ }^{1} \mathrm{H}$ NMR (300 MHz, $\mathrm{CDCl}_{3}$ ): $\delta=3.92-3.87(\mathrm{~m}, 2 \mathrm{H}$ ), 2.77 (br s, $1 \mathrm{H}$ ), 2.50 (br s, $1 \mathrm{H}$ ), 1.75 (t, J = 5.5 Hz, $2 \mathrm{H}$ ), 1.30 (s, $6 \mathrm{H}$ ).

${ }^{13} \mathrm{C}$ NMR $\left(75 \mathrm{MHz}, \mathrm{CDCl}_{3}\right): \delta=71.82,59.94,43.23,29.51$.

MS (ESI, MeOH): $m / z=127[\mathrm{M}+\mathrm{Na}]^{+}$.

HRMS-ESI: $m / z$ [M $+\mathrm{Na}]^{+}$calcd for $\mathrm{C}_{5} \mathrm{H}_{12} \mathrm{NaO}_{2}: 127.0735$; found: 127.0731 .

$\left(1 S^{*}, 5 R^{*}\right)-5-(1-H y d r o x y-1-m e t h y l e t h y l)-2-m e t h y l c y c l o h e x-2-e n-1-$ ol $\left(\mathbf{9}^{\prime}\right)^{21}$

Prepared according to the general procedure from $\mathbf{9}(569 \mathrm{mg})$ and purified by flash column chromatography [silica gel, EtOAc-PE (2:1)] as a colorless oil; yield: $293 \mathrm{mg}(86 \%)$.

${ }^{1} \mathrm{H} \mathrm{NMR}\left(300 \mathrm{MHz}, \mathrm{CDCl}_{3}\right): \delta=5.58(\mathrm{~d}, J=3.6 \mathrm{~Hz}, 1 \mathrm{H}), 4.04(\mathrm{br} \mathrm{s}, 1 \mathrm{H})$, 2.15-2.11 (m, $1 \mathrm{H}), 2.04-2.01(\mathrm{~m}, 1 \mathrm{H}), 1.79(\mathrm{~s}, 3 \mathrm{H}), 1.76-1.69(\mathrm{~m}, 2$ $\mathrm{H}), 1.42(\mathrm{dt}, J=9.9,2.7 \mathrm{~Hz}, 1 \mathrm{H}), 1.22(\mathrm{~s}, 3 \mathrm{H}), 1.89(\mathrm{~s}, 3 \mathrm{H})$.

${ }^{13} \mathrm{C}$ NMR $\left(75 \mathrm{MHz}, \mathrm{CD}_{3} \mathrm{OD}\right): \delta=135.45,126.17,72.79,69.29,39.81$, 34.22, 28.09, 27.21, 27.01, 21.21.

MS (ESI, $\mathrm{MeOH}): m / z=193[\mathrm{M}+\mathrm{Na}]^{+}$.

HRMS-ESI: $m / z$ [M $+\mathrm{Na}]^{+}$calcd for $\mathrm{C}_{10} \mathrm{H}_{18} \mathrm{NaO}_{2}$ : 193.1204; found: 193.1206.

\section{4-Allyl-2-methoxyphenol $\left(\mathbf{1 0}^{\prime}\right)^{29}$}

$\mathrm{NaAuCl}_{4} \cdot 2 \mathrm{H}_{2} \mathrm{O}$ (39.8 mg, $0.1 \mathrm{mmol}, 0.05$ equiv) was added to a solution of silyl ether $\mathbf{1 0}^{30}(557 \mathrm{mg}, 2 \mathrm{mmol})$ in $\mathrm{MeOH}(4 \mathrm{~mL})$ at r.t., and the mixture was stirred at r.t. for $7 \mathrm{~h}$. The mixture was then diluted with EtOAc $(10 \mathrm{~mL})$, filtered through activated alumina, and concentrated in vacuo. The residue was purified by flash column chromatography [silica gel, EtOAc-PE (1:5)] to give a pale-yellow oil; yield: 286 $\mathrm{mg}(87 \%)$.
${ }^{1} \mathrm{H}$ NMR $\left(300 \mathrm{MHz}, \mathrm{CDCl}_{3}\right): \delta=6.86-6.83(\mathrm{~m}, 1 \mathrm{H}), 6.69-6.67(\mathrm{~m}, 2 \mathrm{H})$, 5.99-5.88 (m, $1 \mathrm{H}), 5.49$ (s, $1 \mathrm{H}), 5.10-5.04$ (m, $2 \mathrm{H}$ ), 3.87 (s, $3 \mathrm{H}), 3.32$ (d, $J=6.6 \mathrm{~Hz}, 2 \mathrm{H})$.

${ }^{13} \mathrm{C}$ NMR $\left(75 \mathrm{MHz}, \mathrm{CDCl}_{3}\right): \delta=146.44,143.92,137.80,131.89,121.17$, $115.45,114.26,111.14,55.83,39.84$.

MS (ESI, MeOH): $m / z=187[\mathrm{M}+\mathrm{Na}]^{+}$.

HRMS-ESI: $m / z[\mathrm{M}+\mathrm{Na}]^{+}$calcd for $\mathrm{C}_{10} \mathrm{H}_{12} \mathrm{NaO}_{2}$ : 187.0735; found: 187.0735

\section{4-tert-Butylphenol (11') $)^{4 \mathrm{i}}$}

$\mathrm{NaAuCl}_{4} \cdot 2 \mathrm{H}_{2} \mathrm{O}$ (39.8 mg, $0.1 \mathrm{mmol}, 0.05$ equiv) was added to a solution of silyl ether $\mathbf{1 1}^{4 \mathrm{i}}(529 \mathrm{mg}, 2 \mathrm{mmol})$ in $\mathrm{MeOH}(4 \mathrm{~mL})$ at r.t., and the mixture was stirred at r.t. for $24 \mathrm{~h}$. The mixture was then diluted with EtOAc $(10 \mathrm{~mL})$, filtered through activated alumina, and concentrated in vacuo. The residue was purified by flash column chromatography [silica gel, EtOAc-PE (1:5)] to give a white solid; yield: $277 \mathrm{mg}$ (92\%); mp 97-99 ${ }^{\circ} \mathrm{C}$.

${ }^{1} \mathrm{H}$ NMR $\left(300 \mathrm{MHz}, \mathrm{CDCl}_{3}\right): \delta=7.26(\mathrm{~d}, J=4.5 \mathrm{~Hz}, 2 \mathrm{H}), 6.77(\mathrm{~d}, J=4.5$ $\mathrm{Hz}, 2 \mathrm{H}), 4.65$ (s, $1 \mathrm{H}), 1.29$ (s, $9 \mathrm{H}$ ).

${ }^{13} \mathrm{C}$ NMR (75 MHz, $\left.\mathrm{CDCl}_{3}\right): \delta=153.05,143.58,126.42,114.80,34.05$, 31.51.

MS (ESI, MeOH): $m / z=173[\mathrm{M}+\mathrm{Na}]^{+}$.

HRMS-ESI: $m / z$ [M $+\mathrm{Na}]^{+}$calcd for $\mathrm{C}_{10} \mathrm{H}_{14} \mathrm{NaO}$ : 173.0942 ; found: 173.0354 .

\section{$\mathbf{N}$-(4-Hydroxyphenyl)acetamide $\left(\mathbf{1 2}^{\prime}\right)^{31}$}

$\mathrm{NaAuCl}_{4} \cdot 2 \mathrm{H}_{2} \mathrm{O}$ (39.8 mg, $0.1 \mathrm{mmol}, 0.05$ equiv) was added to a solution of acetamide $12^{32}(531 \mathrm{mg}, 2 \mathrm{mmol})$ in $\mathrm{MeOH}(4 \mathrm{~mL})$ at r.t., and the mixture was stirred at r.t. for $48 \mathrm{~h}$. The mixture was then diluted with EtOAc $(10 \mathrm{~mL})$, filtered through activated alumina, and concentrated in vacuo. The residue was purified by flash column chromatography [silica gel, EtOAc-PE (2:1)] to give a white solid; yield: $260 \mathrm{mg}$ (86\%); mp $166-168^{\circ} \mathrm{C}$.

${ }^{1} \mathrm{H}$ NMR $\left(300 \mathrm{MHz}, \mathrm{CD}_{3} \mathrm{OD}\right): \delta=7.30(\mathrm{~d}, J=6.6 \mathrm{~Hz}, 2 \mathrm{H}), 6.73(\mathrm{~d}, J=6.6$ $\mathrm{Hz}, 2 \mathrm{H}), 2.07$ (s, $3 \mathrm{H})$.

${ }^{13} \mathrm{C}$ NMR $\left(75 \mathrm{MHz}, \mathrm{CD}_{3} \mathrm{OD}\right): \delta=171.46,155.40,131.69,123.54$, 116.28, 23.57.

MS (ESI, MeOH): $m / z=174[\mathrm{M}+\mathrm{Na}]^{+}$.

HRMS-ESI: $m / z[\mathrm{M}+\mathrm{Na}]^{+}$calcd for $\mathrm{C}_{8} \mathrm{H}_{9} \mathrm{NNaO}_{2}: 174.0531$; found: 174.0524 .

\section{1-(4-Hydroxyphenyl)ethanone $\left(\mathbf{1 3}^{\prime}\right)^{8 \mathrm{~b}}$}

$\mathrm{NaAuCl}_{4} \cdot 2 \mathrm{H}_{2} \mathrm{O}$ (79.6 mg, $0.2 \mathrm{mmol}, 0.1$ equiv) was added to a solution of hydroxy ketone $\mathbf{1 3}^{8 \mathrm{~b}}(501 \mathrm{mg}, 2 \mathrm{mmol})$ in $\mathrm{MeOH}(4 \mathrm{~mL})$ at r.t., and the mixture was stirred at r.t. for $48 \mathrm{~h}$. The mixture was then diluted with EtOAc $(10 \mathrm{~mL})$, filtered through activated alumina, and concentrated in vacuo. The residue was purified by flash column chromatography [silica gel, EtOAc-PE (1:5)] to give a white solid; yield: $57 \mathrm{mg}$ (21\%); mp $109-110{ }^{\circ} \mathrm{C}$.

${ }^{1} \mathrm{H}$ NMR $\left(300 \mathrm{MHz}, \mathrm{CDCl}_{3}\right): \delta=7.91(\mathrm{~d}, J=8.7 \mathrm{~Hz}, 2 \mathrm{H}), 6.93(\mathrm{~d}, J=8.7$ $\mathrm{Hz}, 2 \mathrm{H}), 2.58$ (s, $3 \mathrm{H})$.

${ }^{13} \mathrm{C}$ NMR $\left(75 \mathrm{MHz}, \mathrm{CD}_{3} \mathrm{OD}\right): \delta=198.89,161.62,131.24,129.41$, 115.59, 26.21 .

MS (ESI, MeOH): $m / z=159[\mathrm{M}+\mathrm{Na}]^{+}$.

HRMS-ESI: $m / z[\mathrm{M}+\mathrm{Na}]^{+}$calcd for $\mathrm{C}_{8} \mathrm{H}_{8} \mathrm{NaO}_{2}$ : 159.0422; found: 159.0415 


\section{6-(Allyloxy)hexan-1-ol (14') $)^{33}$}

Prepared according to the general procedure from $\mathbf{1 4}^{34}$ (545 mg), and purified by flash column chromatography [silica gel, EtOAc-PE (3:1)] as a colorless oil; yield: $301 \mathrm{mg}$ (95\%).

${ }^{1} \mathrm{H}$ NMR (300 MHz, $\mathrm{CDCl}_{3}$ ): $\delta=5.98-5.85(\mathrm{~m}, 1 \mathrm{H}$ ), 5.27 (dd, $J=1.7$, $17.3 \mathrm{~Hz}, 1 \mathrm{H}$ ), 5.17 (dd, $J=1.1,10.4 \mathrm{~Hz}, 1 \mathrm{H}), 3.97(\mathrm{~d}, J=4.2 \mathrm{~Hz}, 2 \mathrm{H})$, $3.65(\mathrm{t}, J=4.1 \mathrm{~Hz}, 2 \mathrm{H}), 3.44(\mathrm{t}, J=5.0 \mathrm{~Hz}, 2 \mathrm{H}), 1.62-1.56(\mathrm{~m}, 4 \mathrm{H})$, $1.41-1.38(\mathrm{~m}, 5 \mathrm{H})$.

${ }^{13} \mathrm{C}$ NMR $\left(75 \mathrm{MHz}, \mathrm{CDCl}_{3}\right): \delta=134.93,116.64,71.69,70.24,62.64$, $32.56,29.58,25.89,25.51$.

MS (ESI, $\mathrm{MeOH}): m / z=181[\mathrm{M}+\mathrm{Na}]^{+}$.

HRMS-ESI: $m / z[\mathrm{M}+\mathrm{Na}]^{+}$calcd for $\mathrm{C}_{9} \mathrm{H}_{18} \mathrm{NaO}_{2}: 181.1204$; found: 181.1198.

\section{6-Hydroxyhexyl Acetate $\left(\mathbf{1 5}^{\prime}\right)^{4 \mathrm{n}}$}

Prepared according to the general procedure from $15^{4 \mathrm{n}}$ (549 $\mathrm{mg}$ ), and purified by flash column chromatography [silica gel, EtOAc-PE (1:1)] as a colorless oil; yield: $301 \mathrm{mg}(94 \%)$.

${ }^{1} \mathrm{H}$ NMR $\left(300 \mathrm{MHz}, \mathrm{CDCl}_{3}\right): \delta=4.06(\mathrm{t}, J=5.0 \mathrm{~Hz}, 2 \mathrm{H}), 3.64(\mathrm{t}, J=4.8$ $\mathrm{Hz}, 2 \mathrm{H}), 2.05$ (s, $3 \mathrm{H}), 1.64-1.58$ (m, $4 \mathrm{H}), 1.40-1.38$ (m, $4 \mathrm{H})$.

${ }^{13} \mathrm{C}$ NMR $\left(75 \mathrm{MHz}, \mathrm{CDCl}_{3}\right): \delta=171.20,64.40,62.59,32.49,28.49$, 25.63, 25.31, 20.87.

MS (ESI, MeOH): $m / z=183[\mathrm{M}+\mathrm{Na}]^{+}$.

HRMS-ESI: $m / z[M+N a]^{+}$calcd for $\mathrm{C}_{8} \mathrm{H}_{16} \mathrm{NaO}_{3}$ : 183.0997; found: 183.0990.

\section{6-(Methoxymethoxy)hexan-1-ol (16' $)^{4 j}$}

Prepared according to the general procedure from $\mathbf{1 6}^{4 \mathrm{j}}$ (553 $\left.\mathrm{mg}\right)$, and purified by flash column chromatography [silica gel, EtOAc-PE (1:2)] as a colorless oil; yield: $315 \mathrm{mg}$ (97\%).

${ }^{1} \mathrm{H} \mathrm{NMR}\left(300 \mathrm{MHz}, \mathrm{CDCl}_{3}\right): \delta=4.62(\mathrm{~s}, 2 \mathrm{H}), 3.65(\mathrm{t}, J=6.5 \mathrm{~Hz}, 2 \mathrm{H})$, $3.53(\mathrm{t}, J=6.5 \mathrm{~Hz}, 2 \mathrm{H}), 3.36$ (s, $3 \mathrm{H}), 1.75-1.52(\mathrm{~m}, 4 \mathrm{H}), 1.42-1.39(\mathrm{~m}$, $4 \mathrm{H})$.

${ }^{13} \mathrm{C} \mathrm{NMR}\left(75 \mathrm{MHz}, \mathrm{CDCl}_{3}\right): \delta=96.38,67.72,62.84,55.06,32.67,29.66$, 25.99, 25.53 .

MS (ESI, $\mathrm{MeOH}): m / z=185[\mathrm{M}+\mathrm{Na}]^{+}$.

HRMS-ESI: $m / z$ [M $+\mathrm{Na}]^{+}$calcd for $\mathrm{C}_{8} \mathrm{H}_{18} \mathrm{NaO}_{3}: 185.1154$; found: 185.1148 .

\section{6-[(2-Methoxyethoxy)methoxy]hexan-1-ol (17' $)^{4 j}$}

Prepared according to the general procedure from $17^{4 \mathrm{j}}$ (641 mg), and purified by flash column chromatography [silica gel, EtOAc-PE (1:3)] as a colorless oil; yield: $392 \mathrm{mg}(95 \%)$.

${ }^{1} \mathrm{H} \mathrm{NMR}\left(300 \mathrm{MHz}, \mathrm{CDCl}_{3}\right): \delta=4.71(\mathrm{~s}, 2 \mathrm{H}), 3.75-3.62(\mathrm{~m}, 4 \mathrm{H}), 3.60-$ 3.51 (m, $4 \mathrm{H}), 3.40$ (s, $3 \mathrm{H}), 1.75-1.53(\mathrm{~m}, 4 \mathrm{H}), 1.46-1.39(\mathrm{~m}, 4 \mathrm{H})$.

${ }^{13} \mathrm{C} \mathrm{NMR}\left(75 \mathrm{MHz}, \mathrm{CDCl}_{3}\right): \delta=95.43,71.80,67.77,66.68,62.79,58.95$, $32.65,29.59,25.95,25.49$.

MS (ESI, MeOH): $m / z=229[\mathrm{M}+\mathrm{Na}]^{+}$.

HRMS-ESI: $m / z$ [M $+\mathrm{Na}]^{+}$calcd for $\mathrm{C}_{10} \mathrm{H}_{22} \mathrm{NaO}_{4}$ : 229.1416; found: 229.1413.

\section{1,2:3,4-Bis-O-(1-Methylethylidene)- $\alpha$-D-galactopyranose $\left(1^{\prime}\right)^{4 \mathrm{k}}$}

Prepared according to the general procedure from $\mathbf{1 8}^{4 \mathrm{k}}(749 \mathrm{mg})$, and purified by flash column chromatography [silica gel, EtOAc-PE (2:1)] as a colorless oil; yield: $500 \mathrm{mg}(96 \%)$.
${ }^{1} \mathrm{H}$ NMR $\left(300 \mathrm{MHz}, \mathrm{CDCl}_{3}\right): \delta=5.57(\mathrm{~d}, J=5.0 \mathrm{~Hz}, 1 \mathrm{H}), 4.62(\mathrm{dd}, J=7.9$, $2.2 \mathrm{~Hz}, 1 \mathrm{H}), 4.34(\mathrm{~m}, 1 \mathrm{H}), 4.29(\mathrm{t}, J=6.3 \mathrm{~Hz}, 1 \mathrm{H}), 3.95-3.83(\mathrm{~m}, 2 \mathrm{H})$, 3.79-3.72 (m, $1 \mathrm{H}), 2.10(\mathrm{dd}, J=9.6,3.0 \mathrm{~Hz}, 1 \mathrm{H}), 1.54(\mathrm{~s}, 3 \mathrm{H}), 1.46(\mathrm{~s}$, $3 \mathrm{H}), 1.34(\mathrm{~s}, 6 \mathrm{H})$.

${ }^{13} \mathrm{C}$ NMR $\left(75 \mathrm{MHz}, \mathrm{CDCl}_{3}\right)$ : $\delta=109.20,108.45,96.10,71.22,70.57$, $70.43,68.17,61.79,25.82,25.75,24.76,24.18$.

MS (ESI, MeOH): $m / z=283[\mathrm{M}+\mathrm{Na}]^{+}$.

HRMS-ESI: $m / z[\mathrm{M}+\mathrm{Na}]^{+}$calcd for $\mathrm{C}_{12} \mathrm{H}_{20} \mathrm{NaO}_{6}$ : 283.1158; found: 283.1153 .

\section{6-\{[tert-Butyl(diphenyl)silyl]oxy\}hexan-1-ol (19') $)^{4 j}$}

Prepared according to the general procedure from $19^{4 \mathrm{j}}$ (942 mg), and purified by flash column chromatography [silica gel, EtOAc-PE (1:4)] as a colorless oil; yield: $685 \mathrm{mg}$ (96\%).

${ }^{1} \mathrm{H} \mathrm{NMR}\left(300 \mathrm{MHz}, \mathrm{CDCl}_{3}\right): \delta=7.67$ (dd, $\left.J=7.6,1.7 \mathrm{~Hz}, 4 \mathrm{H}\right), 7.45-7.35$ (m, $6 \mathrm{H}), 3.68-3.60(\mathrm{~m}, 4 \mathrm{H}), 1.60-1.53(\mathrm{~m}, 4 \mathrm{H}), 1.36-1.33(\mathrm{~m}, 4 \mathrm{H})$, $1.05(\mathrm{~s}, 9 \mathrm{H})$.

${ }^{13} \mathrm{C}$ NMR $\left(75 \mathrm{MHz}, \mathrm{CDCl}_{3}\right): \delta=135.58,134.16,129.49,127.57,63.85$, 62.98, 32.76, 32.49, 26.88, 25.58, 25.46, 19.22 .

MS (ESI, MeOH): $m / z=379[\mathrm{M}+\mathrm{Na}]^{+}$.

HRMS-ESI: $m / z[\mathrm{M}+\mathrm{Na}]^{+}$calcd for $\mathrm{C}_{22} \mathrm{H}_{32} \mathrm{NaO}_{2} \mathrm{Si}$ : 379.2069; found: 379.2070 .

\section{[4-(\{[tert-Butyl(diphenyl)silyl]oxy\}methyl)phenyl]methanol $\left(\mathbf{2 0}^{\prime}\right)^{35}$}

Prepared according to the general procedure from $\mathbf{2 0}^{35}$ (982 $\mathrm{mg}$ ), and purified by flash column chromatography [silica gel, EtOAc-PE (1:4)] as a colorless oil; yield: $731 \mathrm{mg}(97 \%)$.

${ }^{1} \mathrm{H}$ NMR (300 MHz, $\mathrm{CDCl}_{3}$ ): $\delta=7.72-7.69(\mathrm{~m}, 4 \mathrm{H}), 7.44-7.35(\mathrm{~m}, 10$ $\mathrm{H}), 4.78(\mathrm{~s}, 2 \mathrm{H}), 4.70(\mathrm{~s}, 2 \mathrm{H}), 1.10(\mathrm{~s}, 9 \mathrm{H})$.

${ }^{13} \mathrm{C}$ NMR $\left(75 \mathrm{MHz}, \mathrm{CDCl}_{3}\right.$ ): $\delta=140.61,139.50,135.57,133.53,129.69$, 127.71, 126.99, 126.26, 65.35, 65.29, 26.84, 19.31 .

MS (ESI, MeOH): $m / z=399[\mathrm{M}+\mathrm{Na}]^{+}$.

HRMS-ESI: $m / z$ [M $+\mathrm{Na}]^{+}$calcd for $\mathrm{C}_{24} \mathrm{H}_{28} \mathrm{NaO}_{2} \mathrm{Si}$ : 399.1756; found: 399.1753 .

\section{6-[(Triisopropylsilyl)oxy]hexan-1-ol (21' $)^{4 j}$}

Prepared according to the general procedure from $21^{4 \mathrm{j}}(777 \mathrm{mg})$, and purified by flash column chromatography [silica gel, EtOAc-PE (1:3)] as a colorless oil; yield: $455 \mathrm{mg}(83 \%)$.

$\left.{ }^{1} \mathrm{H} \mathrm{NMR} \mathrm{(300} \mathrm{MHz,} \mathrm{CDCl}_{3}\right): \delta=3.69-3.63(\mathrm{~m}, 4 \mathrm{H}), 1.60-1.54(\mathrm{~m}, 4 \mathrm{H})$, $1.40-1.36$ (m, $4 \mathrm{H}), 1.23$ (br s, $1 \mathrm{H}), 1.11-0.96(\mathrm{~m}, 21 \mathrm{H})$.

${ }^{13} \mathrm{C}$ NMR $\left(75 \mathrm{MHz}, \mathrm{CDCl}_{3}\right): \delta=63.34,62.92,32.94,32.77,25.64,25.56$, 17.99, 12.02.

MS (ESI, MeOH): $m / z=297[\mathrm{M}+\mathrm{Na}]^{+}$.

HRMS-ESI: $m / z$ [M $+\mathrm{Na}]^{+}$calcd for $\mathrm{C}_{15} \mathrm{H}_{34} \mathrm{NaO}_{2} \mathrm{Si}$ : 297.2226; found: 297.2225.

\section{(4-\{[(Triisopropylsilyl)oxy]methyl $\}$ phenyl)methanol (22' $)^{8 \mathrm{e}}$}

Prepared according to the general procedure from $\mathbf{2 2}^{8 \mathrm{e}}$ (818 $\mathrm{mg}$ ), and purified by flash column chromatography [silica gel, EtOAc-PE (1:3)] as a colorless oil; yield: $507 \mathrm{mg}(86 \%)$.

${ }^{1} \mathrm{H}$ NMR $\left(300 \mathrm{MHz}, \mathrm{CDCl}_{3}\right): \delta=7.35-7.34(\mathrm{~m}, 4 \mathrm{H}), 4.84(\mathrm{~s}, 2 \mathrm{H}), 4.68$ (s, $2 \mathrm{H}), 1.60$ (br s, $1 \mathrm{H}), 1.18-1.15$ (m, $3 \mathrm{H}), 1.10-1.06$ (m, $18 \mathrm{H})$.

${ }^{13} \mathrm{C}$ NMR $\left(75 \mathrm{MHz}, \mathrm{CDCl}_{3}\right): \delta=141.20,139.37,126.94,125.96,65.24$, $64.84,18.02,12.05$ 
MS (ESI, $\mathrm{MeOH}): m / z=317[\mathrm{M}+\mathrm{Na}]^{+}$.

HRMS-ESI: $m / z$ [M $+\mathrm{Na}]^{+}$calcd for $\mathrm{C}_{17} \mathrm{H}_{30} \mathrm{NaO}_{2} \mathrm{Si}$ : 317.1913; found: 317.1912 .

\section{[4-(\{[tert-Butyl(dimethyl)silyl]oxy\}methyl)phenyl]methanol $\left(\mathbf{2 3}^{\prime}\right)^{36}$}

$\mathrm{NaAuCl}_{4} \cdot 2 \mathrm{H}_{2} \mathrm{O}(0.8 \mathrm{mg}, 0.002 \mathrm{mmol}, 0.001$ equiv) was added to a solution of disilyl ether $\mathbf{2 3}^{36}$ (733 mg, $2 \mathrm{mmol}$ ) in $\mathrm{MeOH}(4 \mathrm{~mL})$ at r.t., and the mixture was stirred at r.t. for $0.5 \mathrm{~h}$. The mixture was then diluted with EtOAc $(10 \mathrm{~mL})$, filtered through activated alumina, and concentrated in vacuo. The residue was purified by flash column chromatography [silica gel, EtOAc-PE (1:5)] to give a colorless oil; yield: $435 \mathrm{mg}$ (86\%).

${ }^{1} \mathrm{H}$ NMR $\left(300 \mathrm{MHz}, \mathrm{CDCl}_{3}\right): \delta=7.33(\mathrm{~s}, 4 \mathrm{H}), 4.74(\mathrm{~s}, 2 \mathrm{H}), 4.68(\mathrm{~s}, 2 \mathrm{H})$, $0.94(\mathrm{~s}, 9 \mathrm{H}), 0.10(\mathrm{~s}, 6 \mathrm{H})$.

${ }^{13} \mathrm{C}$ NMR $\left(75 \mathrm{MHz}, \mathrm{CDCl}_{3}\right): \delta=140.88,139.54,126.94,126.26,65.13$, $64.77,25.93,18.39,-5.27$.

MS (ESI, MeOH): $m / z=275[\mathrm{M}+\mathrm{Na}]^{+}$.

HRMS-ESI: $m / z$ [M $+\mathrm{Na}]^{+}$calcd for $\mathrm{C}_{14} \mathrm{H}_{24} \mathrm{NaO}_{2} \mathrm{Si}$ : 275.1443; found: 275.1447.

\section{( $)$-trans-[4-(\{[tert-Butyl(dimethyl)silyl]oxy\}methyl)cyclohex- yl]methanol (6) ${ }^{26}$}

$\mathrm{NaAuCl}_{4} \cdot 2 \mathrm{H}_{2} \mathrm{O}(0.8 \mathrm{mg}, 0.002 \mathrm{mmol}, 0.001$ equiv) was added to a solution of disilyl ether $\mathbf{2 4}(745 \mathrm{mg}, 2 \mathrm{mmol})$ in $\mathrm{MeOH}(4 \mathrm{~mL})$ at r.t., and the mixture was stirred at r.t. for $1 \mathrm{~h}$. The mixture was then diluted with EtOAc $(10 \mathrm{~mL})$, filtered through activated alumina, and concentrated in vacuo. The residue was purified by flash column chromatography [silica gel, EtOAc-PE (1:5)] to give a colorless oil; yield: 397 mg (77\%).

${ }^{1} \mathrm{H}$ NMR $\left(300 \mathrm{MHz}, \mathrm{CDCl}_{3}\right): \delta=3.46(\mathrm{~d}, J=4.8 \mathrm{~Hz}, 2 \mathrm{H}), 3.41(\mathrm{~d}, J=4.5$ $\mathrm{Hz}, 2 \mathrm{H}), 1.82-1.80(\mathrm{~m}, 4 \mathrm{H}), 1.44-1.42(\mathrm{~m}, 2 \mathrm{H}), 0.96-0.92(\mathrm{~m}, 4 \mathrm{H})$, $0.89(\mathrm{~s}, 9 \mathrm{H}), 0.04(\mathrm{~s}, 6 \mathrm{H})$.

${ }^{13} \mathrm{C}$ NMR $\left(75 \mathrm{MHz}, \mathrm{CDCl}_{3}\right): \delta=68.70,68.66,40.73,40.61,29.00,28.89$, $25.95,18.36,-5.37$.

MS (ESI, MeOH): $m / z=281[\mathrm{M}+\mathrm{Na}]^{+}$.

HRMS-ESI: $m / z[\mathrm{M}+\mathrm{Na}]^{+}$calcd for $\mathrm{C}_{14} \mathrm{H}_{30} \mathrm{NaO}_{2} \mathrm{Si}$ : 281.1913; found: 281.1919.

\section{(4-\{[tert-Butyl(dimethyl)silyl]oxy\}phenyl)methanol (25' $)^{4 j}$}

Prepared according to the general procedure from $25^{4 \mathrm{j}}$ (705 mg), and purified by flash column chromatography [silica gel, EtOAc-PE (1:3)] as a colorless oil; yield: $439 \mathrm{mg}(92 \%)$.

${ }^{1} \mathrm{H}$ NMR $\left(300 \mathrm{MHz}, \mathrm{CDCl}_{3}\right): \delta=7.22(\mathrm{~d}, J=8.4 \mathrm{~Hz}, 2 \mathrm{H}), 6.82(\mathrm{~d}, J=8.4$ $\mathrm{Hz}, 2 \mathrm{H}), 4.58$ (d, J = 5.7 Hz, $2 \mathrm{H}), 1.76(\mathrm{t}, J=5.8 \mathrm{~Hz}, 1 \mathrm{H}), 0.98(\mathrm{~s}, 9 \mathrm{H})$, $0.19(\mathrm{~s}, 6 \mathrm{H})$.

${ }^{13} \mathrm{C}$ NMR $\left(75 \mathrm{MHz}, \mathrm{CDCl}_{3}\right): \delta=155.31,133.73,128.52,120.15,65.10$, 25.67, $18.20,-4.44$.

MS (ESI, $\mathrm{MeOH}): m / z=261[\mathrm{M}+\mathrm{Na}]^{+}$.

HRMS-ESI: $m / z[\mathrm{M}+\mathrm{Na}]^{+}$calcd for $\mathrm{C}_{13} \mathrm{H}_{22} \mathrm{NaO}_{2} \mathrm{Si}$ : 261.1287; found: 261.1285 .

(2S)-2-((3aR,4S,7aR)-4-\{[tert-butyl(dimethyl)silyl]oxy\}-7a-methyloctahydro-1H-inden-1-yl)propan-1-ol (26') ${ }^{37}$

Prepared according to the general procedure from $\mathbf{2 6}^{37}$ (882 $\mathrm{mg}$ ), and purified by flash column chromatography [silica gel, EtOAc-PE (1:5)] as a colorless oil; yield: $608 \mathrm{mg}(93 \%)$.
${ }^{1} \mathrm{H}$ NMR (300 MHz, $\left.\mathrm{CDCl}_{3}\right): \delta=4.01($ br s, $1 \mathrm{H}), 3.63(\mathrm{dd}, J=3.0,10.2$ $\mathrm{Hz}, 1 \mathrm{H}$ ), 3.37 (dd, $J=6.6,10.2 \mathrm{~Hz}, 1 \mathrm{H}), 1.95$ (d, $J=12.4 \mathrm{~Hz}, 1 \mathrm{H}), 1.87-$ $1.73(\mathrm{~m}, 2 \mathrm{H}), 1.67(\mathrm{dr} \mathrm{s}, 1 \mathrm{H}), 1.63-1.49(\mathrm{~m}, 2 \mathrm{H}), 1.47-1.30(\mathrm{~m}, 4 \mathrm{H})$, $1.27-1.23(\mathrm{~m}, 1 \mathrm{H}), 1.22-1.09(\mathrm{~m}, 3 \mathrm{H}), 1.02(\mathrm{~d}, J=6.6 \mathrm{~Hz}, 3 \mathrm{H}), 0.93(\mathrm{~s}$, $3 \mathrm{H}), 0.89(\mathrm{~s}, 9 \mathrm{H}), 0.01(\mathrm{~s}, 3 \mathrm{H}), 0.00(\mathrm{~s}, 3 \mathrm{H})$.

${ }^{13} \mathrm{C}$ NMR $\left(75 \mathrm{MHz}, \mathrm{CDCl}_{3}\right): \delta=69.34,67.76,53.16,52.83,42.12,40.58$, $38.27,34.41,26.77,25.78,23.10,17.97,17.61,16.66,13.74,-4.84$, -5.19 .

MS (ESI, MeOH): $m / z=349[\mathrm{M}+\mathrm{Na}]^{+}$.

HRMS-ESI: $m / z$ [M $+\mathrm{Na}]^{+}$calcd for $\mathrm{C}_{19} \mathrm{H}_{38} \mathrm{NaO}_{2} \mathrm{Si}$ : 349.2539; found: 349.2535 .

(士)-trans-5-(1-\{[tert-Butyl(dimethyl)silyl]oxy\}-1-methylethyl)-2methylcyclohex-2-en-1-ol (28')

Prepared according to the general procedure from $\mathbf{2 8}(798 \mathrm{mg})$, and purified by flash column chromatography [silica gel, EtOAc-PE (1:5)] as a colorless oil; yield: $489 \mathrm{mg}$ (86\%).

IR (KBr): 3350, 2969, 2922, 2857, 1468, 1379, $1250 \mathrm{~cm}^{-1}$.

${ }^{1} \mathrm{H}$ NMR $\left(300 \mathrm{MHz}, \mathrm{CDCl}_{3}\right): \delta=5.57-5.56(\mathrm{~m}, 1 \mathrm{H}), 4.01(\mathrm{br} \mathrm{s}, 1 \mathrm{H})$, 2.13-2.06 (m, $1 \mathrm{H}), 2.02(\mathrm{dq}, J=2.0,13.6, \mathrm{~Hz}, 1 \mathrm{H}), 1.86-1.81(\mathrm{~m}, 1 \mathrm{H})$, $1.78(\mathrm{~s}, 3 \mathrm{H}), 1.61$ (ddt, $J=2.4,4.8,12.0 \mathrm{~Hz}, 1 \mathrm{H}), 1.43(\mathrm{dt}, J=4.0,13.6$ Hz, 2 H), 1.21 (s, 3 H), 1.19 (s, 3 H), 0.85 (s, 9 H), 0.07 (s, $6 \mathrm{H}$ ).

${ }^{13} \mathrm{C}$ NMR $\left(75 \mathrm{MHz}, \mathrm{CDCl}_{3}\right): \delta=134.16,125.86,74.70,69.00,39.84$, $32.88,27.80,27.75,26.93,25.93,20.83,18.28,-2.03$.

MS (ESI, MeOH): $m / z=307[\mathrm{M}+\mathrm{Na}]^{+}$.

HRMS-ESI: $m / z$ [M $+\mathrm{Na}]^{+}$calcd for $\mathrm{C}_{16} \mathrm{H}_{32} \mathrm{NaO}_{2} \mathrm{Si}$ : 307.2069; found: 307.2083.

\section{(3,4,5-Trimethoxyphenyl)methanol $\left(\mathbf{3 0}^{\prime}\right)^{22}$}

Prepared according to the general procedure from $\mathbf{3 0}(625 \mathrm{mg})$, and purified by flash column chromatography [silica gel, EtOAc-PE (1:5)] as a white solid; yield: $361 \mathrm{mg}(91 \%) ; \mathrm{mp} 36-38^{\circ} \mathrm{C}$.

$\left.{ }^{1} \mathrm{H} \mathrm{NMR} \mathrm{(300} \mathrm{MHz,} \mathrm{CDCl}_{3}\right): \delta=6.60(\mathrm{~s}, 2 \mathrm{H}), 4.63(\mathrm{~s}, 2 \mathrm{H}), 3.87(\mathrm{~s}, 6 \mathrm{H})$, $3.84(\mathrm{~s}, 3 \mathrm{H}), 1.81$ (br s, $1 \mathrm{H})$.

${ }^{13} \mathrm{C}$ NMR $\left(75 \mathrm{MHz}, \mathrm{CDCl}_{3}\right): \delta=153.24,137.16,136.68,103.72,65.32$, $60.75,55.98$.

MS (ESI, MeOH): $m / z=221[\mathrm{M}+\mathrm{Na}]^{+}$.

HRMS-ESI: $m / z[M+N a]^{+}$calcd for $\mathrm{C}_{10} \mathrm{H}_{14} \mathrm{NaO}_{4}$ : 221.0790; found: 221.0792 .

\section{1-(\{[6-(Benzyloxy)hexyl]oxy\}methyl)-4-methoxybenzene (29) ${ }^{38}$}

$\mathrm{NaAuCl}_{4} \cdot 2 \mathrm{H}_{2} \mathrm{O}$ (39.8 mg, $0.1 \mathrm{mmol}, 0.05$ equiv) was added to a solution of silyl ether 1 ( $645 \mathrm{mg}, 2 \mathrm{mmol}$ ) and 4- $\mathrm{MeOC}_{6} \mathrm{H}_{4} \mathrm{CH}_{2} \mathrm{OH}(3)$ (1.24 $\mathrm{mL}, 10 \mathrm{mmol})$ in THF ( $4 \mathrm{~mL}$ ) and the mixture was refluxed for $8 \mathrm{~h}$. The mixture was then cooled to r.t., diluted with EtOAc $(10 \mathrm{~mL})$, filtered through activated alumina, and concentrated in vacuo. The residue was purified by flash column chromatography [silica gel, EtOAc-PE (1:10)] to give a colorless oil; yield: $394 \mathrm{mg}$ (60\%).

${ }^{1} \mathrm{H} \mathrm{NMR}\left(300 \mathrm{MHz}, \mathrm{CDCl}_{3}\right): \delta=7.33-7.35(\mathrm{~m}, 5 \mathrm{H}), 7.27(\mathrm{~d}, J=8.4 \mathrm{~Hz}, 2$ $\mathrm{H}), 6.90(\mathrm{~d}, J=8.4 \mathrm{~Hz}, 2 \mathrm{H}), 4.52(\mathrm{~s}, 2 \mathrm{H}), 4.45(\mathrm{~s}, 2 \mathrm{H}), 3.83(\mathrm{~s}, 3 \mathrm{H})$, 3.50-3.44 (m, $4 \mathrm{H}), 1.63-1.58$ ( $\mathrm{m}, 4 \mathrm{H}), 1.41-1.39$ (m, $4 \mathrm{H})$.

${ }^{13} \mathrm{C} \mathrm{NMR}\left(75 \mathrm{MHz}, \mathrm{CDCl}_{3}\right): \delta=159.06,138.66,130.76,129.13,128.27$, 127.54, 127.39, 113.70, 72.79, 72.45, 70.35, 70.04, 55.19, 29.67, 26.01.

MS (ESI, MeOH): $m / z=351[\mathrm{M}+\mathrm{Na}]^{+}$.

HRMS-ESI: $m / z[\mathrm{M}+\mathrm{Na}]^{+}$calcd for $\mathrm{C}_{21} \mathrm{H}_{28} \mathrm{NaO}_{3}: 351.1936$; found: 351.1939 . 


\section{1,2,3-Trimethoxy-5-(methoxymethyl)benzene (31) ${ }^{39}$}

$\mathrm{NaAuCl}_{4} \cdot 2 \mathrm{H}_{2} \mathrm{O}$ (39.8 mg, $0.1 \mathrm{mmol}, 0.05$ equiv) was added to a solution of silyl ether 30 (624 mg, $2 \mathrm{mmol}$ ) in $\mathrm{MeOH}(4 \mathrm{~mL})$, and the mixture was refluxed for $24 \mathrm{~h}$. The mixture was then cooled to r.t., diluted with EtOAc $(10 \mathrm{~mL})$, filtered through activated alumina, and concentrated in vacuo. The residue was purified by flash column chromatography [silica gel, EtOAc-PE (1:10)] to give a colorless oil; yield: $266 \mathrm{mg}$ (63\%).

$\left.{ }^{1} \mathrm{H} \mathrm{NMR} \mathrm{(300} \mathrm{MHz,} \mathrm{CDCl}_{3}\right): \delta=6.57(\mathrm{~s}, 2 \mathrm{H}), 4.39$ (s, $\left.2 \mathrm{H}\right), 3.87(\mathrm{~s}, 6 \mathrm{H})$, $3.84(\mathrm{~s}, 3 \mathrm{H}), 3.41(\mathrm{~s}, 3 \mathrm{H})$.

${ }^{13} \mathrm{C}$ NMR $\left(75 \mathrm{MHz}, \mathrm{CDCl}_{3}\right): \delta=153.26,137.46,133.85,104.61,74.85$, 60.76, 58.11, 56.05.

MS (ESI, $\mathrm{MeOH}): m / z=235[\mathrm{M}+\mathrm{Na}]^{+}$.

HRMS-ESI: $m / z[\mathrm{M}+\mathrm{Na}]^{+}$calcd for $\mathrm{C}_{11} \mathrm{H}_{16} \mathrm{NaO}_{4}$ : 235.0946; found: 235.0948 .

\section{Acknowledgment}

This work was supported by the National Natural Science Foundation of China (21302119), the Research Fund for the Doctoral Program of Higher Education of China (20100202120002), and the Fundamental Research Funds for the Central Universities (GK201302017).

\section{References}

(1) Greene, T. W.; Wuts, P. G. M. Protective Groups in Organic Synthesis, 3rd ed.; Wiley: New York, 1999.

(2) Kocienski, P. J. Protecting Groups, 3rd ed.; Thieme: Stuttgart, 2005.

(3) For a recent review, see: Crouch, R. D. Tetrahedron 2013, 69, 2383; and references therein.

(4) For recent references, see: (a) González-Calderó, D.; BenitezPuebla, L. J.; Gonzalez-Gonzalez, C. A.; Garcia-Eleno, M. A.; Fuentes-Benitez, A.; Cuevas-Yañez, E.; Corona-Becerril, D.; González-Romero, C. Synth. Commun. 2014, 44, 1258. (b) Jadhav, A. H.; Chinnappan, A.; Patil, R. H.; Chung, W.-J.; Kim, H. Chem. Eng. J. (Amsterdam, Neth.) 2014, 236, 300. (c) Bothwell, J. M.; Angeles, V. V.; Carolan, J. P.; Olson, M. E.; Mohan, R. S. Tetrahedron Lett. 2010, 51, 1056. (d) Martinez-Solorio, D.; Jennings, M. P. Tetrahedron Lett. 2008, 49, 5175. (e) Bhure, M. H.; Kumar, I.; Natu, A. D.; Rode, C. V. Synth. Commun. 2008, 38, 346. (f) Shah, S. T. A.; Guiry, P. J. Org. Biomol. Chem. 2008, 6, 2168. (g) Yang, Y.-Q.; Cui, J.-R.; Zhu, L.-G.; Sun, Y.-P.; Wu, Y. Synlett 2006, 1260. (h) Das, B.; Reddy, K. R.; Thirupathi, P. Tetrahedron Lett. 2006, 47, 5855. (i) Bhatt, S.; Nayak, S. K. Tetrahedron Lett. 2006, 47, 8395. (j) Yeom, C.-E.; Kim, Y. J.; Lee, S. Y.; Shin, Y. J.; Kim, B. M. Tetrahedron 2005, 61, 12227. (k) Kumar, G. D. K.; Baskaran, S. J. Org. Chem. 2005, 70, 4520. (l) Karimi, B.; Zareyee, D. Tetrahedron Lett. 2005, 46, 4661. (m) Hua, J.; Jiang, Z. Y.; Wang, Y. G. Chin. Chem. Lett. 2004, 15, 1430. (n) Rani, S.; Babu, J. L.; Vankar, Y. D. Synth. Commun. 2003, 33, 4043. (o) Yadav, J. S.; Reddy, B. V. S.; Madan, C. New J. Chem. 2000, 24, 853.

(5) (a) Yeom, C.-E.; Kim, H. W.; Lee, S. Y.; Kim, B. M. Synlett 2007, 146. (b) Jiang, Z.-Y.; Wang, Y.-G. Chem. Lett. 2003, 32, 568. (c) Jiang, Z.-Y.; Wang, Y.-G. Tetrahedron Lett. 2003, 44, 3859. (d) Crouch, R. D.; Stieff, M.; Frie, J. L.; Cadwallader, A. B.; Bevis, D. C. Tetrahedron Lett. 1999, 40, 3133. (e) Wilson, N. S.; Keay, B. A. Tetrahedron Lett. 1997, 38, 187.
(6) (a) Patel, P.; Chang, C.-T.; Kang, N.; Lee, G.-J.; Powell, W. S.; Rokach, J. Tetrahedron Lett. 2007, 48, 5289. (b) Ikawa, T.; Hattori, K.; Sajiki, H.; Hirota, K. Tetrahedron 2004, 60, 6901. (c) Kim, S.; Jacobo, S. M.; Chang, C.-T.; Bellone, S.; Powell, W.; Rokach, J. Tetrahedron Lett. 2004, 45, 1973. (d) Corey, E. J.; Jones, G. B. J. Org. Chem. 1992, 57, 1028. (e) de Vries, E. F. J.; Brussee, J.; van der Gen, A. J. Org. Chem. 1994, 59, 7133. (f) Cormier, J. F. Tetrahedron Lett. 1991, 32, 187.

(7) (a) Li, J.; Menche, D. Synthesis 2009, 1904. (b) Reddy, M. S.; Narender, M.; Nageswar, Y. V. D.; Rao, K. R. Synthesis 2005, 714. (c) Karimi, B.; Zamani, A.; Zareyee, D. Tetrahedron Lett. 2004, 45, 9139. (d) Wang, M.; Li, C.; Yin, D.; Liang, X.-T. Tetrahedron Lett. 2002, 43, 8727. (e) Sabitha, G.; Syamala, M.; Yadav, J. S. Org. Lett. 1999, 1, 1701. (f) DattaGupta, A.; Singh, R.; Singh, V. K. Synlett 1996, 69. (g) Oku, A.; Kinugasa, M.; Kamada, T. Chem. Lett. 1993, 165.

(8) (a) Shah, S. T. A.; Singh, S.; Guiry, P. J. J. Org. Chem. 2009, 74, 2179. (b) Ranu, B. C.; Jana, U.; Majee, A. Tetrahedron Lett. 1999, 40, 1985. (c) Zhang, W.; Robins, M. J. Tetrahedron Lett. 1992, 33, 1177. (d) Corey, E. J.; Yi, K. Y. Tetrahedron Lett. 1992, 33, 2289. (e) Pilcher, A. S.; Hill, D. K.; Shimshock, S. J.; Waltermire, R. E.; DeShong, P. J. Org. Chem. 1992, 57, 2492. (f) Metcalf, B. W.; Burkhart, J. P.; Jund, K. Tetrahedron Lett. 1980, 21, 35. (g) Corey, E. J.; Venkateswarlu, A. J. Am. Chem. Soc. 1972, 94, 6190.

(9) (a) Jadhav, A. H.; Kim, H. Tetrahedron Lett. 2012, 53, 5338. (b) Yan, L.; Zhao, F.; Gan, Y.; Zhao, J.; Jiang, Z. Synth. Commun. 2012, 42, 285. (c) Wang, B.; Sun, H.-X.; Sun, Z.-H. J. Org. Chem. 2009, 74, 1781. (d) Itoh, A.; Kodama, T.; Masaki, Y. Chem. Pharm. Bull. 2007, 55, 861. (e) Iida, A.; Okazaki, H.; Misaki, T.; Sunagawa, M.; Sasaki, A.; Tanabe, Y.J. Org. Chem. 2006, 71, 5380. (f) Khan, A. T.; Ghosh, S.; Choudhury, L. H. Eur. J. Org. Chem. 2004, 2198. (g) Zubaidha, P. K.; Bhosale, S. V.; Hashmi, A. M. Tetrahedron Lett. 2002, 43, 7277. (h) Bartoli, G.; Cupone, G.; Dalpozzo, R.; De Nino, A.; Maiuolo, L.; Procopio, A.; Sambri, L.; Tagarelli, A. Tetrahedron Lett. 2002, 43, 5945. (i) Tandon, M.; Begley, T. P. Synth. Commun. 1997, 27, 2953. (j) Maiti, G.; Roy, S. C. Tetrahedron Lett. 1997, 38, 495. (k) Lee, A. S.-Y.; Yeh, H.-C.; Tsai, M.-H. Tetrahedron Lett. 1995, 36, 6891.

(10) Arcadi, A.; Pietropaolo, E.; Alvino, A.; Michelet, V. Org. Lett. 2013, 15, 2766.

(11) Xu, M.; Hou, Q.; Wang, S.; Wang, H.; Yao, Z.-J. Synthesis 2011, 626.

(12) Yan, B.; Liu, Y. Org. Lett. 2007, 9, 4323.

(13) Alfonsi, M.; Arcadi, A.; Aschi, M.; Bianchi, G.; Marinelli, F. J. Org. Chem. 2005, 70, 2265.

(14) Georgy, M.; Boucard, V.; Debleds, O.; Zotto, C. D.; Campagne, J.-M. Tetrahedron 2009, 65, 1758.

(15) Georgy, M.; Boucard, V.; Campagne, J.-M. J. Am. Chem. Soc. 2005, $127,14180$.

(16) Cuenca, A.; Mancha, G.; Asensio, G.; Medio-Simón, M. Chem. Eur. J. 2008, 14, 1518.

(17) Jiang, C.; Wang, S. Synlett 2009, 1099.

(18) Terrasson, V.; Marque, S.; Georgy, M.; Campagne, J.-M.; Prim, D. Adv. Synth. Catal. 2006, 348, 2063.

(19) Collington, E. W.; Finch, H.; Smith, I. J. Tetrahedron Lett. 1985, 26,681 .

(20) Lee, A. S.-Y.; Yeh, H.-C.; Yeh, M.-K.; Tsai, M.-H.J. Chin. Chem. Soc. (Taipei) 1995, 42, 919.

(21) da Silva Rocha, K. A.; Hoehne, J. L.; Gusevskaya, E. V. Chem. Eur. J. 2008, 14, 6166.

(22) Baumert, M.; Albrecht, M.; Winkler, H. D. F.; Schalley, C. A. Synthesis 2010, 953. 
(23) Zhang, L.; Wang, S.; Zhou, S.; Yang, G.; Sheng, E. J. Org. Chem. 2006, 71, 3149.

(24) Davies, H. M. L.; Hedley, S. J.; Bohall, B. R. J. Org. Chem. 2005, 70, 10737.

(25) Hardcastle, I. R.; Liu, J.; Valeur, E.; Watson, A.; Ahmed, S. U.; Blackburn, T. J.; Bennaceur, K.; Clegg, W.; Drummond, C.; Endicott, J. A.; Golding, B. T.; Griffin, R. J.; Gruber, J.; Haggerty, K.; Harrington, R. W.; Hutton, C.; Kemp, S.; Lu, X.; McDonnell, J. M.; Newell, D. R.; Noble, M. E. M.; Payne, S. L.; Revill, C. H.; Riedinger, C.; Xu, Q.; Lunec, J. J. Med. Chem. 2011, 54, 1233.

(26) Li, P.; Yamamoto, H. Chem. Commun. 2009, 5412.

(27) Marković, D.; Steunenberg, P.; Ekstrand, M.; Vogel, P. Chem. Commun. 2004, 2444.

(28) Johnson, D. A.; Taubner, L. M. Tetrahedron Lett. 1996, 37, 605.

(29) Elgendy, E. M.; Khayyat, S. A. Russ. J. Org. Chem. 2008, 44, 823.

(30) Ohmiya, H.; Tanabe, M.; Sawamura, M. Org. Lett. 2011, 13, 1086.
(31) Lewis, E. A.; Adamek, T. L.; Vining, L. C.; White, R. L. J. Nat. Prod. 2003, 66, 62.

(32) Mai, K.; Patil, G. J. Org. Chem. 1986, 51, 3545.

(33) Inagaki, M.; Hiratake, J.; Yamamoto, Y.; Oda, J. Bull. Chem. Soc. Jpn. 1987, 60, 4121.

(34) Reddy, C. S.; Smitha, G.; Chandrasekhar, S. Tetrahedron Lett. 2003, 44, 4693.

(35) Zhang, S.; Xu, L.; Trudell, M. L. Synthesis 2005, 1757.

(36) Itoh, A.; Kodama, T.; Masaki, Y. Synlett 1999, 357.

(37) Fraga, R.; Zacconi, F.; Sussman, F.; Ordóñez-Morán, P.; Muñoz, A.; Huet, T.; Molnár, F.; Moras, D.; Rochel, N.; Maestro, M.; Mouriño, A. Chem. Eur. J. 2012, 18, 603.

(38) Shen, Z.; Sheng, L.; Zhang, X.; Mo, W.; Hu, B.; Sun, N.; Hu, X. Tetrahedron Lett. 2013, 54, 1579.

(39) Ohira, S.; Shirane, F.; Nozaki, H.; Yahiro, S.; Nakayama, M. Bull. Chem. Soc. Jpn. 1989, 62, 2427.

This article differs from the e-first online version only in its layout; no content has been changed. 